\title{
PUblications Metadata Augmentation (PUMA) pipeline
}

\section{[version 1; peer review: 2 approved with reservations]}

\author{
Oliver W. Butters (iD)1-3, Rebecca C. Wilson (iD)1-3, Hugh Garner (iD2,3, \\ Thomas W. Y. Burton (D) 3,4
}

\footnotetext{
${ }^{1}$ Department of Public Health, Policy and Systems, University of Liverpool, Liverpool, UK

${ }^{2}$ Population Health Sciences Institute, Newcastle University, UK, Newcastle upon Tyne, UK

${ }^{3}$ Social and Community Medicine, University of Bristol, Bristol, UK

${ }^{4}$ Department of Computer Science, University of Oxford, Oxford, UK
}

V1 First published: 04 Sep 2020, 9:1095
https://doi.org/10.12688/f1000research.25484.1

Latest published: 12 Apr 2021, 9:1095

https://doi.org/10.12688/f1000research.25484.2

\section{Abstract}

Cohort studies collect, generate and distribute data over long periods of time - often over the lifecourse of their participants. It is common for these studies to host a list of publications (which can number many thousands) on their website to demonstrate the impact of the study and facilitate the search of existing research to which the study data has contributed. The ability to search and explore these publication lists varies greatly between studies.

We believe a lack of rich search and exploration functionality is a barrier to entry for new or prospective users of a study's data, since it may be difficult to find and evaluate previous work in a given area. These lists of publications are also typically manually curated, resulting in a lack of rich metadata to analyse, making bibliometric analysis difficult.

We present here a software pipeline that aggregates metadata from a variety of third-party providers to power a web based search and exploration tool for lists of publications. Alongside core publication metadata (i.e. author lists, keywords etc.), we include geocoding of first authors and citations in our pipeline. This allows a characterisation of a study as a whole based on common locations of authors, frequency of keywords, citation profile etc. This enriched publications metadata can be useful for generating project impact metrics and web-based graphics useful for public dissemination. In addition, the pipeline produces a research data set for bibliometric analysis or social studies of science.

Keywords

Longitudinal birth cohort, Bibliography, Bibliometrics, ALSPAC

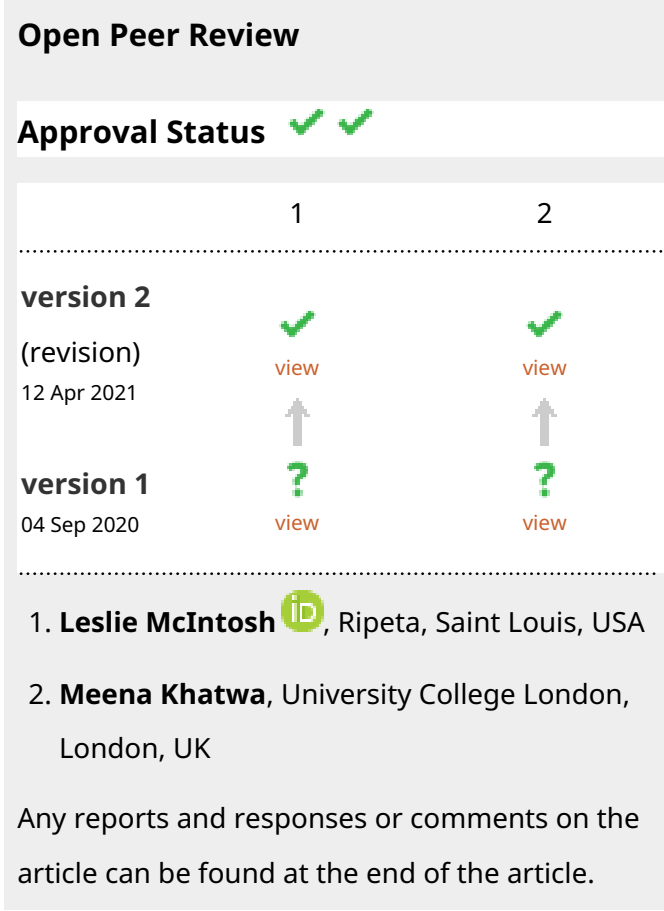


This article is included in the Research on

Research, Policy \& Culture gateway.

Corresponding author: Oliver W. Butters (olly.butters@liverpool.ac.uk)

Author roles: Butters OW: Conceptualization, Funding Acquisition, Methodology, Project Administration, Software, Writing - Original Draft Preparation, Writing - Review \& Editing; Wilson RC: Conceptualization, Funding Acquisition, Methodology, Project Administration, Software, Supervision, Writing - Original Draft Preparation, Writing - Review \& Editing; Garner H: Methodology, Software, Writing Review \& Editing; Burton TWY: Software, Writing - Review \& Editing

Competing interests: No competing interests were disclosed.

Grant information: This project was primarily funded by CLOSER, whose mission is to maximise the use, value and impact of longitudinal studies. CLOSER is funded by the Economic and Social Research Council (ESRC) and Medical Research Council (MRC) (grant reference: ES/K000357/1). The funders took no role in the design, execution, analysis or interpretation of the data or in the writing up of the findings. www.closer.ac.uk The UK Medical Research Council and Wellcome (Grant ref: 102215/2/13/2) and the University of Bristol provide core support for ALSPAC. This publication is the work of the authors they will serve as guarantors for the contents of this article. RW is a UKRI Innovation Fellow with HDR UK [MR/S003959/1]. Additional support was provided by: The Wellcome Trust and Medical Research Council (grant number 108439/Z/15/Z). The European Union's Horizon 2020 research and innovation programme under grant agreement No 824989 and the Canadian Institutes of Health Research (CIHR). The National Institute for Health Research Applied Research Collaboration. The views expressed in this publication are those of the author(s) and not necessarily those of the National Institute for Health Research or the Department of Health and Social Care.

The funders had no role in study design, data collection and analysis, decision to publish, or preparation of the manuscript.

Copyright: $\odot 2020$ Butters OW et al. This is an open access article distributed under the terms of the Creative Commons Attribution License, which permits unrestricted use, distribution, and reproduction in any medium, provided the original work is properly cited.

How to cite this article: Butters OW, Wilson RC, Garner $\mathrm{H}$ and Burton TWY. PUblications Metadata Augmentation (PUMA) pipeline [version 1; peer review: 2 approved with reservations] F1000Research 2020, 9:1095 https://doi.org/10.12688/f1000research.25484.1 First published: 04 Sep 2020, 9:1095 https://doi.org/10.12688/f1000research.25484.1 


\section{Introduction}

Cohort studies collect, generate and distribute huge amounts of longitudinal data for health, social and economic research based on a defined group of people over an extended period of time (often many years). Birth cohort studies begin at birth (or sometimes before) and often continue over the course of their participants' entire lifetime. The UK is home to many cohort studies and several birth cohort studies, including some that have been running for decades (e.g. the National Survey of Health and Development (NSHD), which started in 1946 ${ }^{1}$ ).

Typically researchers can apply for, and access, these data sets once various relevant governance conditions have been met $^{2}$.

Studies often keep track of the publications that have arisen from the data they have given to researchers for project monitoring purposes and to report back to their funder(s). The length of these lists of publications is sometimes used as a crude metric of the research outputs or impact for the study.

The CLOSER (Cohort \& Longitudinal Studies Enhancement Resources) consortium (https://www.closer.ac.uk) comprises eight UK birth cohort studies and is used here as an illustration of typical birth cohort studies. Each of the studies holds a list of their publications on their respective public facing websites. The specific purpose, functionality and user interface of these lists varies from study to study. Some studies have publications lists that are comprised exclusively of peer-reviewed journal articles, others have a much broader remit and include a variety of other written outputs, e.g. books, reports, conference proceedings, media examples etc. The way this data is presented varies greatly, ranging from downloadable static PDF files, through static lists on web pages split by year, to interactive web pages.

The search functionality of each study also varies greatly, with the static page approach offering no other way to search than a browser-based free text search on the rendered text available on a given page. Where only a subset of publications is shown (e.g. if it is split by year), or where rich metadata is missing (e.g. if no keyword or abstract text is available/rendered), it is difficult or impossible to search for given terms. Some studies have a web form which allows a free text search on a database across author, journal, title and abstract text, split by year. One study has an advanced searching capability letting users search on author, subject, article type, as well as free text searching on title, abstract etc. None of the CLOSER studies have any kind of metrics kept alongside their publication lists, e.g. citations.

We consider the lack of a comprehensive publication search and exploration facility a barrier to entry for researchers unfamiliar with a study. When presented with potentially thousands of publications it can be difficult to find existing publications in a given research area, and when relevant publications have been found, lack of usage information (e.g. in the form of download statistics, citation counts etc) can make it difficult to prioritise reading lists. It is possible that some potential users of a study's data fall at this first hurdle and do not proceed with an application for data to the study. This could have an effect on the overall impact of the study (since less new research is done) and in the case of studies that charge for data, it will have a direct financial implication. A similar scenario may occur if a researcher doesn't find a relevant publication and applies for data to carry out a project that has already been conducted.

In addition to the difficulty of searching and exploring data, the lack of good metadata makes it impossible to do bibliometric analysis on a study's publications. This means questions such as 'where are all the first authors based?' or 'are there trends in subject areas over time?' can only be addressed either anecdotally, or with significant manual input (see e.g. 3).

This article describes an open source software pipeline (PUMA -PUblications Metadata Augmentation pipeline) which takes a list of publications and augments it with metadata from a selection of third-party metadata providers. This augmented metadata set has two distinct use cases: 1) bibliometric analysis and 2) providing a web based searching and exploration tool. Examples of the potential bibliometric analyses possible with this augmented metadata include: calculating the total number of citations a study has generated, characterising a study based on the keywords of its publications, highlighting the geographic or institutional distribution of first authors, the variety of authors, assessing which journals are published in most frequently, how each of these metrics is changing over time, as well as a variety of other uses. We demonstrate some of these bibliometric uses and a web based exploration tool based on the augmented metadata set provided by PUMA in this article.

While the exemplar publications list used here is a U.K. birth cohort, this pipeline could be applied to almost any research study or project that has a list of publications with a rich set of persistent identifiers, particularly in the biomedical domain.

\section{Existing tools}

There are several well established bibliography management tools in which users can manually curate their own bibliographies and easily use them to add formatted references to their written work (see https://en.wikipedia.org/wiki/Comparison_of_ reference_management_software for a reasonable list). These include proprietary tools such as EndNote and Mendeley, as well as open source tools like Zotero. A common feature among them is to automatically incorporate available publication metadata from an external source (such as Web of Science, Scopus, CrossRef and others) into each bibliography item. The wide variety and differing levels of completeness of available metadata means that typically a core set of fields are used (e.g. author list is common, but funding information is not). Also, static fields tend to be used, so an author list is common but a citation count is not. These subsets of all available metadata can typically be exported from the various tools in a variety of formats (e.g. BibTeX, RIS etc). There is little focus on gaining insight from the bibliographies in these software packages beyond grouping by keywords/themes. 
The big three bibliometric metadata hubs (Web of Science, Google Scholar and Scopus) all have web based accounts which allow the curation of lists of journal articles and keeps track of the number of citations each article has. They also offer some basic citation analytics such as h-indexes and i10-indexes.

The focus of these bibliographic tools (both the online hubs and the software) is for an individual's own published works, or an individual's collection of publications which they may want to reference later on. Inbuilt to most of the tools is an automatic publication suggestion mechanism which uses the metadata of existing publications to suggest other publications based on common attributes (e.g. similar author lists or keywords).

There are several other projects which focus on specific visualisation or analytics of existing metadata sets. SurVis ${ }^{4}$ creates an interactive web based exploration tool based on a static set of BibTeX metadata files. This allows filtering by author or keywords that exist in the static metadata files.

Network analysis of authors, subjects, journals, keywords and citations is another area of development, with tools such as CiteWiz $^{5}$, PivotSlice ${ }^{6}$ and VOS Viewer ${ }^{7}$ featuring analysis and visualistion of clusters, trends over time and in depth querying mechanisms.

These bibliography management, visualisation and analysis tools variously allow the curation of bibliographies, assist in finding similar articles, and give some insight to static metadata. No single existing tool gives easy access to aggregated and processed non-static metadata from a variety of sources to enable both in depth bibliographic study as well as providing an easy to use (potentially public facing) mechanism to explore publication metadata of a long running study.

\section{Persistent identifiers}

Modern academic journal articles are typically assigned persistent identifiers when they are published. The aim of these is to give a consistent and long lasting mechanism to refer to them. Often a journal will assign a unique journal-specific identifier to an article which resolves to the article on the journal's website. In addition to this, a Digital Object Identifier (DOI) is usually assigned. DOIs are the de facto persistent identifier used across the academic journal publishing sector.

DOI resolving services exist to refer users (human and machine) to the relevant journal web page. These resolving services also host a wealth of metadata themselves. The service used to resolve DOIs in this work is the canonical resolver: doi.org (see e.g. the DOI data model -https://www.doi.org/doi_handbook/4_ Data_Model.html).

In addition to doi.org there exist other resolving and metadata services that are domain-specific. These may have more in depth and domain specific metadata beyond that offered by the general data model provided by doi.org. In this work we also make use of the persistent identifiers that the National Center for
Biotechnology Information (NCBI) PubMed generates (PubMed IDs-PMID), and the metadata their resolving service provides ${ }^{8}$. This offers us extra metadata over and above that available from doi.org, although on a subset of all available publications.

\section{Exemplar publication list}

The exemplar list of publications considered in this project is from the Avon Longitudinal Study of Parents and Children (ALSPAC - https://www.bristol.ac.uk/alspac). The ALSPAC began in 1990 and as a consequence of this their publications are relatively modern and there is a good coverage of DOIs. The nature of the research done with ALSPAC data is largely biomedical, which gives a high proportion of publications with PMIDs. ALSPAC reports to have over 2000 publications as of April 2019 (http://www.bristol.ac.uk/alspac/news/2019/bristol-families-co90s.html). For this work we use the cleaned BibTeX list of ALSPAC publications ${ }^{9}$ described in 10. For a general overview of ALSPAC see 11.

\section{Methods}

\section{Implementation}

PUMA is built as a pipeline of several discrete stages, the first stage retrieves a list of publications, then subsequent stages add and derive information for each publication in the list before passing it on to the next stage. The end goal of this augmentation stage is a metadata object containing as many metadata items in Table 1 as possible. This is achieved by first retrieving the list of publications from Zotero; adding metadata to it from doi.org, PubMed, and Scopus; geocoding the first author's institute; and getting citation counts. Once this metadata object has been built PUMA can then do some basic statistics and generate HTML pages to allow exploration and searching. This is explained in detail below, and shown in overview in Figure 1.

Zotero. Zotero is an online, free-to-use and open-source bibliography manager. It allows publications metadata to be grouped together into user defined libraries. Here we use it to hold the canonical list of unique publications for a given study (ALSPAC in this exemplar). Zotero allows publications metadata to be entered manually (by filling in the fields by hand), semi-manually (by adding e.g. a DOI and it querying external sources), or programmatically using its API.

Once a publication's metadata is stored in Zotero it can be updated as required. One particularly useful feature of Zotero is its built in deduplication function. Whilst the exemplar list of publications used here is clean (i.e. there are no duplicates in it), if a new list is used it is likely that this will not be the case. Data cleaning can be done in Zotero, and this is made easier by Zotero pulling in metadata from external sources (Crossref and PubMed for the items here). This can highlight, and enables easy fixing of, errors such as where a DOI has been mistyped and points to an incorrect publication.

The PUMA pipeline begins by using the Zotero API (v3) to get the library for the study. This is presented as a series of JSON files (one per publication in Zotero), containing all the metadata 
Table 1. Final metadata object. This is a tabular representation of the python dictionary used to store the metadata, the secondary column items are nested under the primary items where present. Metadata source key: Zr=Zoteroraw, S=Scopus, $D=$ doi.org, $\mathrm{P}=$ PubMed, Ze='Extra' field from Zotero, W=Wikidata De=Derived. The metadata sources are used in the order they are displayed in the table (left to right), once a value has been found the subsequent sources are not queried.

\begin{tabular}{|c|c|c|}
\hline Primary & Secondary & Source \\
\hline \multirow{5}{*}{ IDs } & DOI & $\mathrm{Zr}$ \\
\hline & PMID & Ze \\
\hline & Scopus & $S$ \\
\hline & Hash & De \\
\hline & Zotero & $\mathrm{Zr}$ \\
\hline \multirow{3}{*}{ Authors } & Author list & $P / D$ \\
\hline & First author & Author list/Ze \\
\hline & Affiliation & D/P/S/Ze \\
\hline \multirow{5}{*}{ Location } & Canonical institute & De \\
\hline & Town & W \\
\hline & Country & W \\
\hline & Longitude & W \\
\hline & Latitude & W \\
\hline Date & & $\mathrm{P} / \mathrm{D} / \mathrm{S} / \mathrm{Ze} / \mathrm{Zr}$ \\
\hline Title & & $\mathrm{P} / \mathrm{D} / \mathrm{S} / \mathrm{Zr}$ \\
\hline Abstract & & $\mathrm{P}$ \\
\hline Citations & Scopus citation count & $\mathrm{S}$ \\
\hline \multirow[t]{2}{*}{ Keywords } & $\mathrm{MeSH}$ & $P$ \\
\hline & Other & $\mathrm{P}$ \\
\hline \multirow{3}{*}{ Journal } & Name & $\mathrm{D} / \mathrm{P}$ \\
\hline & Volume & $\mathrm{D} / \mathrm{P}$ \\
\hline & Issue & $\mathrm{D} / \mathrm{P}$ \\
\hline
\end{tabular}

held on a given publication. These are downloaded and cached locally by the pipeline.

While all of the metadata is downloaded from Zotero, the PUMA pipeline disregards most of it as it does not map to the final metadata object well. The most important fields used from the Zotero metadata are the DOI and PMID, since these are the identifiers used to query external metadata providers. DOI has a native field in Zotero, but PMID does not and is stored as a key-value pair in the Zotero 'extra' field. There are a small number of fields where if the metadata is missing from doi.org and PubMed, then the metadata is used from Zotero. Where there is a direct match to the native Zotero data type (e.g. title) that

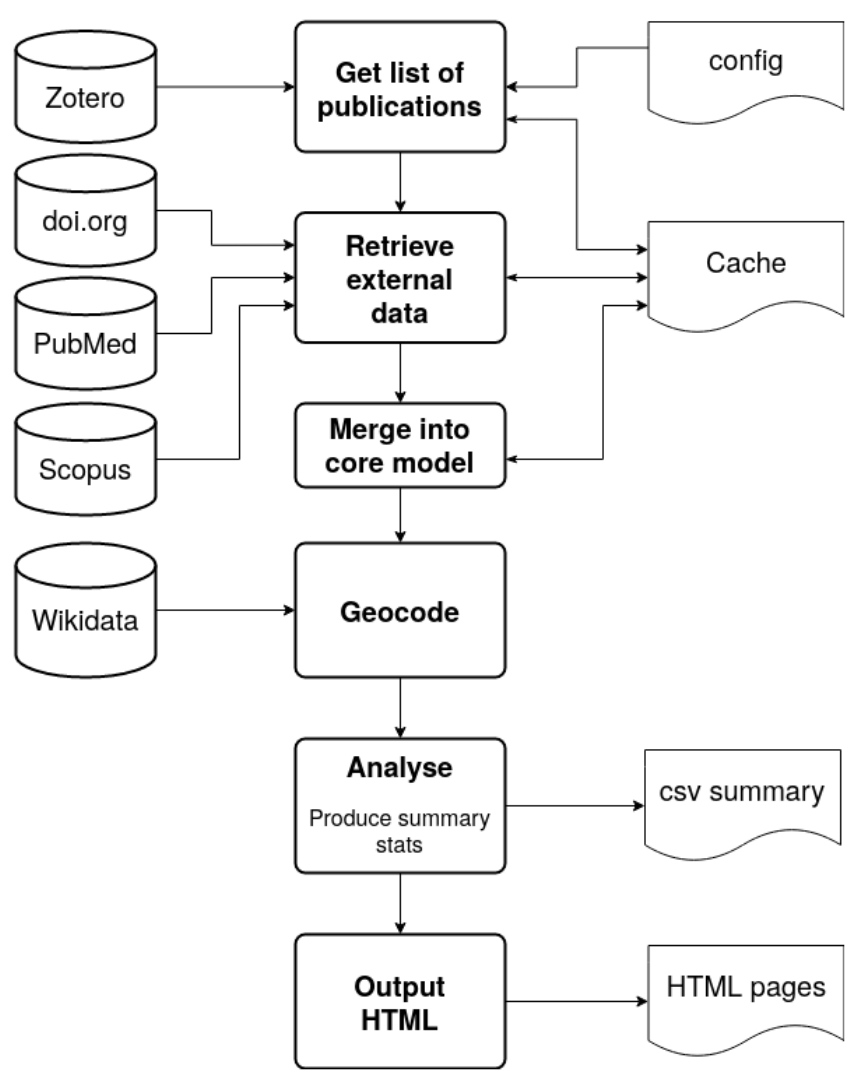

Figure 1. Overview of the PUMA pipeline. The left column shows the sources of data accessed via their APIs, the central column the stages the pipeline with the right column showing input and output of the pipeline.

is used, where there is not a match (e.g. Zotero doesn't have an affiliation field for authors) then a key-value pair is used in the 'extra' field. This is outlined in Table 1.

doi.org. The pipeline then cycles through the list of publications, and where a DOI is present in the metadata from Zotero, it queries the doi.org API with it. If it is a valid DOI then doi.org will return a JSON file containing all the metadata it holds on this publication, which is cached locally.

PubMed central. If the Zotero metadata contains a PMID then the pipeline will then query the PubMed central API to get any extra metadata. The resulting XML file is cached for later use. The metadata available from PubMed is richer than that available via doi.org, including biomedical domain specific fields such as MeSH headings.

Scopus. Scopus is then queried via it's API. The query is first tried with a PMID, then if no value is found the query is repeated using the DOI as the identifier.

The use of Scopus data has some constraints on it depending on the context in which it is used. The most relevant condition 
here is that where citation counts are displayed on a website they must link back to the relevant publication in Scopus, and must be updated at least weekly (https://dev.elsevier.com/tecdoc_ attribution_scopus.html).

Merging DOI, PMID \& Scopus metadata. As noted earlier, the metadata from doi.org and PubMed will contain different fields. Moreover, the same field may have different names in the two sources. In order to merge the metadata in a meaningful way we developed a mapping from each of the relevant fields to what we consider the local canonical version. In some cases our mappings required several fall-backs, e.g. the date of a publication in PubMed has six different places that it could be specified. This is due to a combination of the PubMed schema changing over time, the completeness of the data when it is input into PubMed, and genuine different relevant dates e.g. date published online and data published in print.

The mapping is done into our core set of fields (see Table 1) for each metadata source. Our mapping process initially creates a simple metadata object based on the Zotero ID, DOI and PubMed ID. Into this metadata object it then copies the relevant fields from the DOI, PMID and Scopus metadata.

Geocode. We assume the first author of the publication is the primary author, then we attempt to assign a canonical institute to them. This assignment is done by using a manually built lookup table which initially tries to use the email address of the first author, then if that fails, the postal address. In order to get consistent geographical information of a publication we take a university to be the smallest unit (i.e. two different departments at the same university will not be distinguished in the geocoding). The reason behind this is that there is very little consistency between publications on how department addresses are formatted. The same strategy is used for hospital departments and companies. Our definition of what a canonical institute name is is based on how it appears in wikidata (https://www.wikidata.org).

For the email address based matching we attempt to match exactly the domain part of the email address to a canonical institute (e.g. someone@ncl.ac.uk gets mapped to Newcastle University). Email addresses that are generic or personal (e.g. someone@gmail.com) are ignored.

If there is no matching email address then we attempt to match the postal address. The lookup table has multiple entries for several organisations where authors use non-canonical names e.g. 'Newcastle University' and 'The University of Newcastle' both map to Newcastle University.

Since a publication list may go back many years, there may be institutes that no longer exist (perhaps having been renamed, merged with other institutes or shut down entirely). The lookup table therefore has several entries of now defunct institutes which are mapped to from email addresses and postal addresses.

Once we have the canonical name for an institute we use the wikidata SPARQL API to get the institute's geolocation, town and country (wikidata properties: P625, P131 and P17, respectively). In some cases the first author's institute may be a large multinational or distributed organisation, in which case we use the head quarters location as defined on wikidata (property P159). If any of this data does not exist on wikidata we try to add it.

Data quality. The nature of the manual curation of a list of publications can lead to some missing information and errors. This ranges from there not being any persistent identifiers present, to multiple copies of the same publication being present in different forms, e.g. a preprint and the final version. In order to address these data quality issues we built an interface to assist further cleaning of the metadata, there are two main facets to this interface: highlighting issues and making fixing issues easier.

The interface is a large HTML table with a row for each publication and columns for the status of relevant attributes. Where a value of an attribute is useful in the data cleaning process it is displayed (e.g. DOI and PMID), where the presence of an attribute is more useful than its value (e.g. first author) then just an indication of it's presence is given. Missing attributes are colour coded to make them easy to see, and the table can be sorted by value/presence of attributes. Where, for example, a PMID is missing, the relevant table cell is coloured orange and there is a clickable link which queries PubMed for this publication based on its DOI or title. Similar approaches are available to find DOIs via PubMed and Scopus. Where this provides missing IDs (DOI or PMID) they can be added to Zotero and the pipeline rerun.

Some metadata may not be present in the external providers metadata for some publications -even with the correct DOIs and PMIDs in Zotero. In this case the metadata can be used directly from Zotero for a small number of fields as indicated in Table 1. As with the DOI and PMID case above, the missing metadata will be highlighted orange in the table, and once it has been added to Zotero the pipeline will need to be run again.

There are some derived metadata items that, if missing, will be highlighted in red; this indicates that a setting in the pipeline or a local configuration file is causing the problem. An example would be if a first author institute is found in the source metadata, but there is no matching entry in the institute look up file then a canonical institute cannot be set. The lookup file needs to be updated and the pipeline rerun in this case.

Simple analyses. The pipeline then does some simple processing of the metadata so it can be used for reporting and which feeds into the generated web pages (see below). It outputs (as a CSV file) the frequency of the authors (separately the full author list and first author only), the first author's institute and the journal the publication appeared in.

The keywords, title and abstract text in a publication all serve to give an overview of the content. The keywords are sometimes from a controlled vocabulary, e.g. Medical Subject Headings (MeSH). The titles and abstracts having more free text offer the ability to be more descriptive. From a searching perspective the 
greater freedom with abstracts makes them more searchable/ findable ${ }^{12}$. To derive some meaning from all of the available text in all of the publications from a study, the pipeline calculates the frequency of each word in the keywords, titles, and abstracts. To process the text it converts all text to lower case and removes all punctuation. It then takes out the name of the study, so in this case the exact phrase "Avon Longitudinal Study of Parents and Children", and variations of, but individual components are kept if they were used outside of that context e.g. if 'parents' is used in a different sentence. Then common words such as the, and etc are disregarded. Then the Python Natural Language Tool $\mathrm{Kit}^{13}$ is used to lemmatize each word into its base component. With this clean set of words the pipeline then calculates the frequency of each. It also does this broken down by year, so it is possible to see how trends in research areas change over time in a long running study.

Generated web pages. The pipeline generates static HTML pages which allow the search and exploration of the augmented metadata sets. These pages include filtering by year and by keywords, and visualisations of some of the metadata. The static HTML pages are completely encapsulated, meaning that they can be viewed without the need for a web server etc. As such, PUMA can run locally to generate the data and statistics, and then the HTML files used to explore it.

Figure 2, Figure 3 and Figure 4 show example plots from the generated web pages, with live versions available at https://ollybutters.github.io/puma/alspac/.

\section{Operation}

The pipeline is written in Python 3 and is available from GitHub (https://github.com/OllyButters/puma). Some prerequisite
Python libraries are required to run the PUMA pipeline, these are described on the wiki at https://github.com/OllyButters/ puma/wiki and in the requirements.txt file in the source folder.

The behaviour of the pipeline (which API keys to use, date ranges, colour schemes, caching behaviour etc) is controlled by a configuration file. A sample configuration file is available in the config directory, with guidance on how to populate it at https://github.com/OllyButters/puma/wiki/Configuration. The pipeline has been developed in linux environment, and can be run from the command line by calling the papers.py file in the source directory. This assumes a config.ini file in the config directory, if a different file name is used then it can be specified when running the pipeline with the config option: papers.py --config-file-name.ini. We have also run PUMA in a Windows 10 environment with a minimum of python 3.6.

The pipeline is designed so that it can be rerun regularly, running as a regular CRON job for example. Metadata is cached locally wherever possible, making subsequent pipeline runs much quicker after the initial run. This is possible as the metadata from doi.org and PubMed is very stable, so changes are rare to an individual publication's metadata. The citation counts are cached for as long as is specified in the configuration file, being updated as required.

\section{Use case}

While the intention of this article is to describe the pipeline and the work flow developed to ingest metadata into it we also show some example outputs of the pipeline without interpretation.

We imported the ALSPAC BibTeX data into a new collection in a new group library in Zotero, giving the coverage of fields

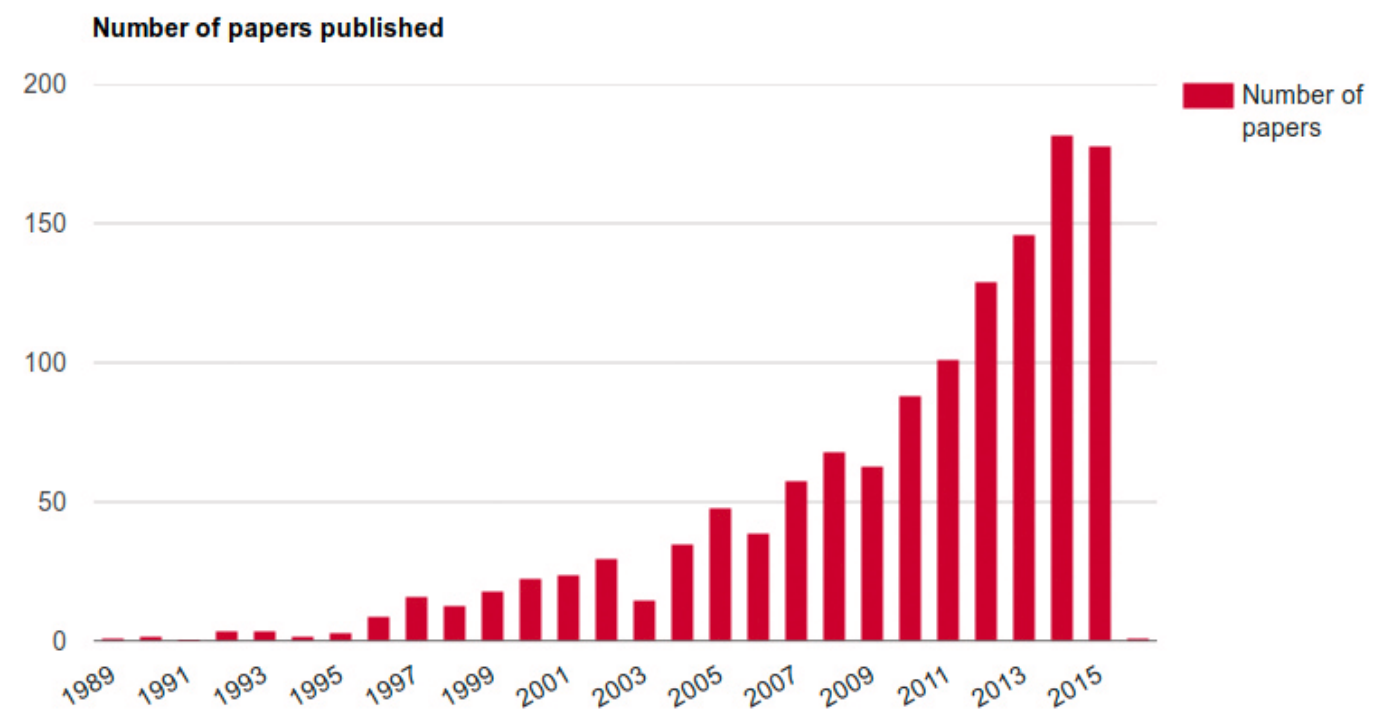

Data from 1,300 publications

Figure 2. Number of publications per year in ALSPAC. 


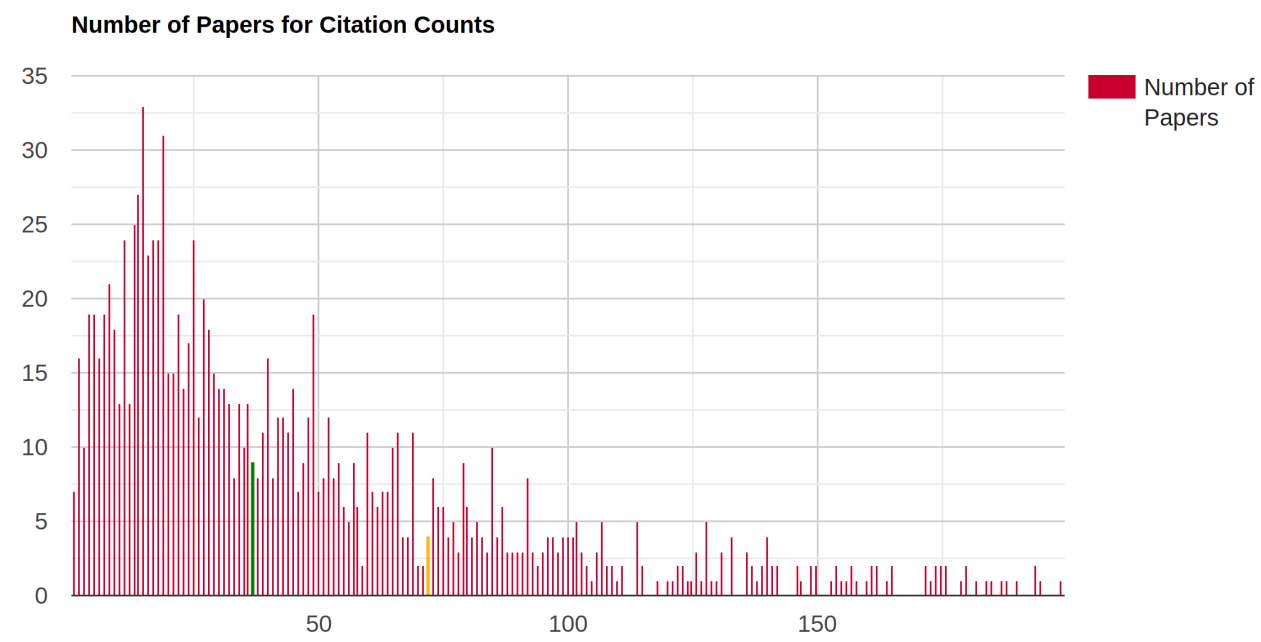

Mean number of citations

Median number of citations

Number of Citations

Data from 1,284 publications

Figure 3. Citation count profile of ALSPAC publications as of $\mathbf{1 5 / 7 / 2 0 2 0}$. The $x$-axis is truncated at 200 citations as there are a small number of publications disparately spread above this.

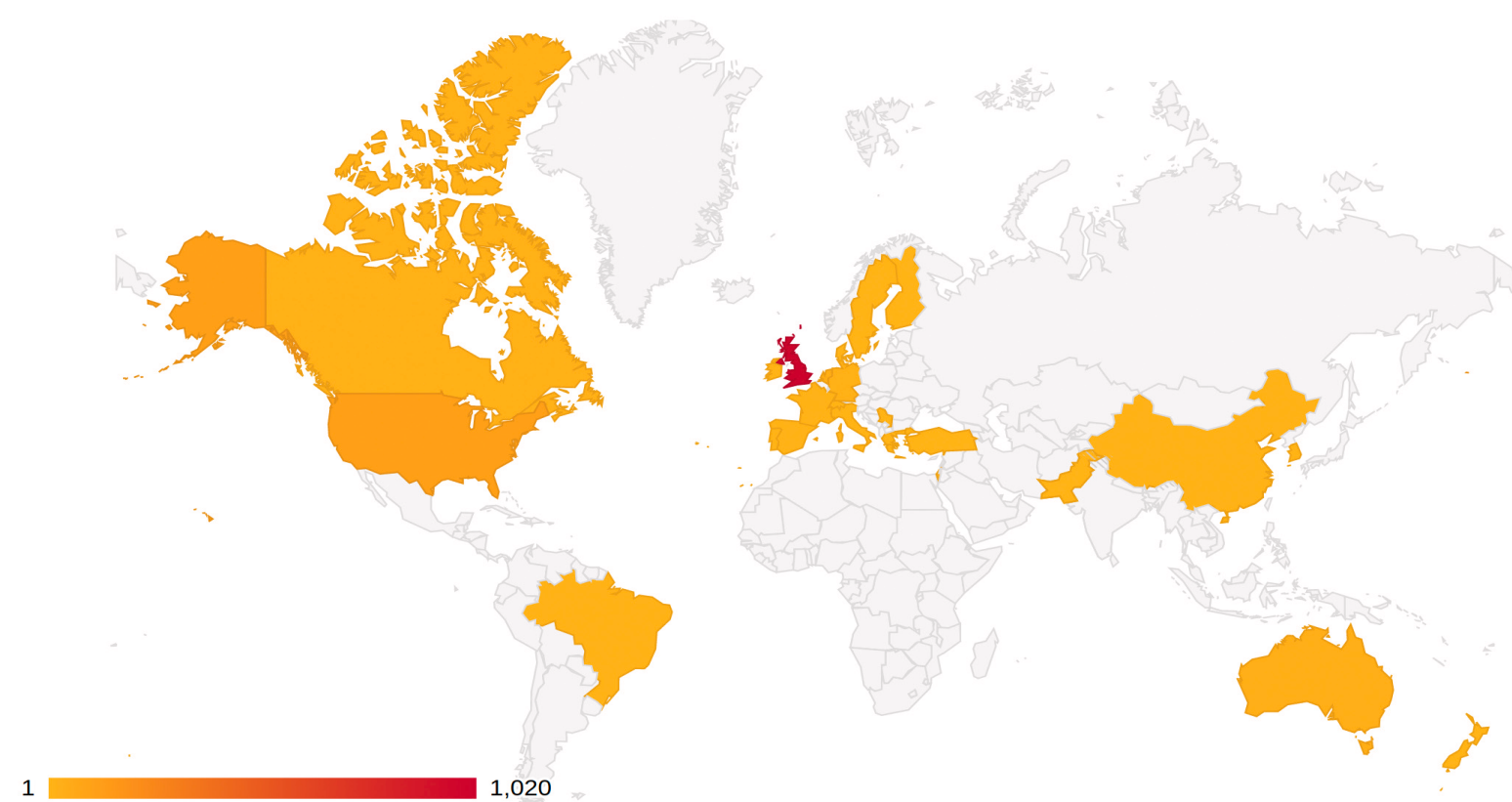

Data from 1,269 publications

Figure 4. Choropleth map of first author countries in ALSPAC.

as outlined in 10. The PUMA pipeline works best with at least one of DOI and PMID for each publication, coverage of these fields in the source metadata is outlined in Table 2. See Underlying data for a list of the references used ${ }^{14}$.

For this initial metadata set the pipeline achieved the augmented metadata coverage outlined in Table 3. Where there are gaps in this metadata it is mostly due to actual missing metadata in the source systems, however the incompleteness in the geocoded metadata is due to a combination of authors using a consortium name as their affiliation, or the metadata containing only a fragment of their address. These could easily be manually addressed with the 'extra' field in Zotero, however since the purpose of this article is to outline the PUMA pipeline and not to strive for a $100 \%$ coverage of the metadata, we have not added any 'extra' metadata to Zotero.

\section{Number of publications per year}

Figure 2 shows the number of publications published per year for ALSPAC from 1989 to 2015. This is the most basic 
Table 2. Source metadata coverage.

\begin{tabular}{|l|r|}
\hline Date range & $1989-2015$ \\
\hline Publication count & 1300 \\
\hline DOIs & 1260 \\
\hline PMIDs & 1240 \\
\hline At least one of DOI or PMID & 1293 \\
\hline
\end{tabular}

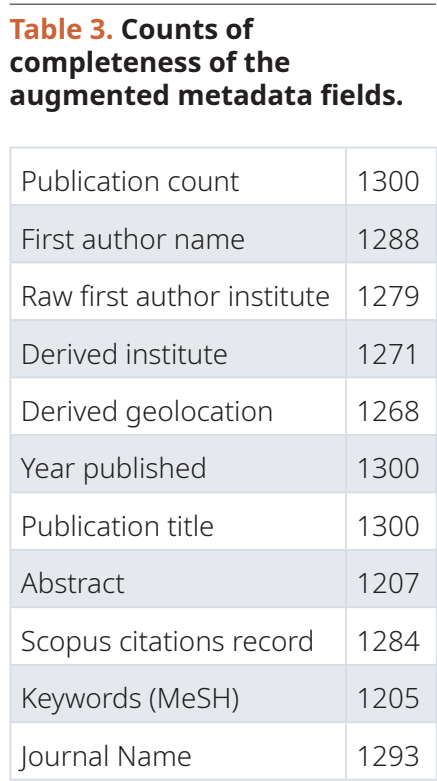

information from the pipeline, and is already information that is easily available to the studies.

\section{Simple citation statistics}

Table 4 shows some basic study level citation calculations. As noted above, the incompleteness of the metadata will impact the numbers here, specifically ALSPAC has 98\% Scopus coverage, meaning all the citation numbers in Table 4 will likely be slightly under-reported.

Figure 3 shows the profile of citation counts for ALSPAC publications with a citation count less than 200 .

\section{Geolocation}

Using the geolocation data generated from the pipeline we can plot a choropleth map of countries first authors are based in. Figure 4 shows the first authors location for $98 \%$ of the publications. Again, this is affected by the coverage of the metadata.

Keywords, titles and abstracts

Table 5 shows the result of a frequency analysis of lemmatized keywords, title text and abstract text of all of the publications that have relevant metadata. This metadata can be further broken down by year to show study changes over time.

\section{Discussion}

\section{Limitations}

One limitation to this work, which is difficult to address, is the completeness of the source list of publications. It is common for cohort studies to ask researchers to inform them when they publish their research based on the study's data. This request is not always complied with, so the source lists of publications are prone to being incomplete. This will have an impact on the insights the PUMA pipeline can generate, with some aspects just under-reporting (e.g. the total citation count) while others may give a misleading picture if there is a systematic reason for the missing publications (e.g. the frequency of keywords in a study will be misleading if all publications from a field are missing).

One of the key assumptions we have made is that the first author is the primary author for the publication. This does vary across different scientific disciplines - it may be that the first author is the one who did the bulk of the work, or that they wrote up the

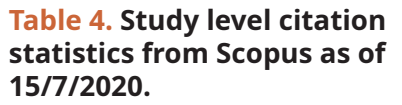

\begin{tabular}{|l|r|}
\hline Number of publications & 1300 \\
\hline Number with citation data & 1284 \\
\hline Total citation count & 93,016 \\
\hline h-index & 137 \\
\hline c100-index & 211 \\
\hline $\begin{array}{l}\text { Mean citations per } \\
\text { publication }\end{array}$ & 72 \\
\hline
\end{tabular}

Table 5. Frequency of top ten lemmatized words used in keywords, titles and abstract text from the ALSPAC publications. The numbers in parentheses are the count.

\begin{tabular}{|l|l|l|}
\hline Keywords & Title & Abstract \\
\hline study (1513) & study (357) & child (2517) \\
\hline child (1284) & child (291) & age (2034) \\
\hline human(1257) & cohort (259) & association (1905) \\
\hline female (1050) & childhood (220) & associated (1696) \\
\hline male (859) & association (220) & study (1675) \\
\hline factor (720) & birth (146) & year (1553) \\
\hline infant (568) & age (129) & risk (1142) \\
\hline longitudinal (562) & risk (128) & maternal (1120) \\
\hline pregnancy (470) & maternal (122) & ci (915) \\
\hline adolescent (470) & associated (117) & cohort (904) \\
\hline
\end{tabular}


majority of the publication, or they just appear first alphabetically. While this will not have an effect on the publication-level statistics (e.g. how many citations it has), it may have an effect on where we have assigned a geographic location.

Linking on author name is also problematic when multiple authors have the same name, or where there are multiple spellings of a given name. This can occur where names have been converted to e.g. ASCII on their way into metadata records. Another instance is where a name is sometimes hyphenated and others not (in this exemplar data set there exists entries for Davey Smith and Davey-Smith). It is important not to place too much emphasis on citations and to not treat them as an exact value. While it is easy to measure how many publications are cited in a single publication, it is difficult to establish the inverse - i.e. how many publications in all the literature cite a given publication. This is due to the completeness of the source literature which is used to calculate the incoming citations, which means that different providers of citation counts will likely give different answers (see 15 and 16).

\section{Future work}

The modular nature of the pipeline means that it is straightforward to add different data sources. One source that we plan to add is Altmetric, which tracks mentions of publications in the media (including social media) and links these back to a DOI.
We also plan to link directly with Crossref (using their API) to pull in a richer set of metadata.

Some of the modern PubMed metadata, and a lot of the Crossref metadata, include information on grants (increasingly with a grant reference code). This would allow us to investigate who the major funders of users of the data are.

\section{Data availability}

Underlying data

Zenodo: ALSPAC peer reviewed publications 1989-2015. http:// doi.org/10.5281/zenodo. $2276785^{14}$.

This project contains the list of publications from the Use case. All other metadata is pulled in from external APIs at run time.

Data are available under the terms of the Creative Commons Attribution 4.0 International license (CC-BY 4.0).

\section{Software availability}

Source code available from: https:/github.com/OllyButters/ puma.

Archived source code at time of publication: http://doi. org/10.5281/10.5281/zenodo.3971102 ${ }^{17}$.

License: GNU General Public License v3.0.
1. Kuh D, Pierce $M$, Adams J, et al: Cohort profile: updating the cohort profile for the MRC National Survey of Health and Development: a new clinicbased data collection for ageing research. Int J Epidemiol. 2011; 40(1): e1-e9. PubMed Abstract | Publisher Full Text | Free Full Text

2. Murtagh MJ, Blell MT, Butters OW, et al.: Better governance, better access: practising responsible data sharing in the METADAC governance infrastructure. Hum Genomics. 2018; 12(1): 24. PubMed Abstract | Publisher Full Text | Free Full Text

3. Barbosa SDJ, Silveira MS, Gasparini I: What publications metadata tell us about the evolution of a scientific community: the case of the Brazilian human-computer interaction conference series. Scientometrics. 2017; 110(1): 275-300.

Publisher Full Text

4. Beck F, Koch S, Weiskopf D: Visual Analysis and Dissemination of Scientific Literature Collections with SurVis. IEEE Trans Vis Comput Graph. 2016; 22(1): 180-189.

PubMed Abstract | Publisher Full Text

5. Elmqvist N, Tsigas P: CiteWiz: A tool for the visualization of scientific citation networks. Inf Vis. 2007; 6(3): 215-232. Publisher Full Text

6. Zhao J, Collins C, Chevalier F, et al.: Interactive Exploration of Implicit and Explicit Relations in Faceted Datasets. IEEE Trans Vis Comput Graph. 2013. 19(12): 2080-2089.

PubMed Abstract | Publisher Full Text

7. van Eck NJ, Waltman L: Software survey: voSviewer, a computer program for bibliometric mapping. Scientometrics. 2010; 84(2): 523-538. PubMed Abstract | Publisher Full Text | Free Full Text

8. NCBI Resource Coordinators: Database resources of the national center for biotechnology information. Nucleic Acids Res. 2018; 46(D1): D8-D13. PubMed Abstract | Publisher Full Text | Free Full Text

9. Butters O, Ismail A, Thompson S, et al.: ALSPAC peer reviewed publications
1989-2015. 2018.

Publisher Full Text

10. Butters O, Ismail A, Thompson S, et al.: Generation of a cleaned dataset listing Avon Longitudinal Study of Parents And Children peer-reviewed publications to 2015 [version 1; peer review: 2 approved]. Wellcome Open Res. 2018; 3: 161.

PubMed Abstract | Publisher Full Text | Free Full Text

11. Boyd A, Golding J, Macleod J, et al.: Cohort Profile: The 'Children of the 90s' - the index offspring of the Avon Longitudinal Study of Parents and Children. Int J Epidemiol. 2013; 42(1): 111-127. PubMed Abstract | Publisher Full Text | Free Full Text

12. Van Kasteren $Y$, Williams $P A H$, Maeder A: Identifying emerging trends in medical informatics: A synthesis approach. Stud Health Technol Inform. 2017. 235: 506-510.

PubMed Abstract | Publisher Full Text

13. Bird S, Loper E, Klein E: Natural Language Processing with Python. O'Reilly Media Inc., 2009. Reference Source

14. Butters O, Ismail A, Thompson S, et al.: ALSPAC peer reviewed publications 1989-2015. 2018.

http://www.doi.org/10.5281/zenodo.2276785

15. Mingers J, Leydesdorff $\mathrm{L}:$ A review of theory and practice in scientometrics. Eur J Oper Res. 2015; 246(1): 1-19. Publisher Full Text

16. Martín-Martín A, Thelwall M, Orduna-Malea E, et al.: Google Scholar, Microsoft Academic, Scopus, Dimensions, Web of Science, and OpenCitations' COCI: a multidisciplinary comparison of coverage via citations. arXiv. 2004.14329, 2020. Reference Source

17. Butters O, Wilson B, Garner H, et al.: OllyButters/puma: v1.2. 2020. http://www.doi.org/10.5281/zenodo.3971102 


\section{Open Peer Review}

\section{Current Peer Review Status: ? ?}

\section{Version 1}

Reviewer Report 16 December 2020

https://doi.org/10.5256/f1000research.28123.r70885

(c) 2020 Khatwa M. This is an open access peer review report distributed under the terms of the Creative Commons Attribution License, which permits unrestricted use, distribution, and reproduction in any medium, provided the original work is properly cited.

\section{Meena Khatwa}

Evidence for Policy and Practice Information and Co-ordinating Centre, UCL Institute of Education, University College London, London, UK

\section{Overall comments}

This was a very interesting article on finding ways to find a user-friendly way to source and manage data.

As a systematic reviewer, the processes used are not so dissimilar to how we scope and search for evidence.

On the whole I found the paper engaging and have only minor suggestions and comments to make. I am a qualitative research who specialised in qualitative evidence synthesis and thematic analysis. Therefore I positioned myself as someone who as a first time researcher would be interested in applying this method.

\section{Abstract}

Clear and concise but I would have made it more explicit this was project to trial/test PUma.

\section{Introduction}

I understand this project was primarily funded by CLOSER; "This project was primarily funded by CLOSER, whose mission is to maximise the use, value and impact of longitudinal studies. CLOSER is funded by the Economic and Social Research Council (ESRC) and Medical Research Council (MRC) (grant)" hence using their data, as you state as an 'illustration' ( $\mathrm{p} 3$ ). It would have useful to have compared to another data set to have avoided any conflict of interest or biasness.

"Some studies have a web form which allows a free text search on a database across author, journal, title and abstract text, split by year. One study has an advanced searching capability letting users search on author, subject, article type, as well as free text searching on title, abstract etc. None of the CLOSER studies have any kind of metrics kept alongside their publication lists, e.g. citations". (p3)

This paragraph needs to make more clarity as it is unclear, what do you mean by one study and what is that study. The information is a bit vague. 
"We consider the lack of a comprehensive publication search and exploration facility a barrier to entry for researchers unfamiliar with a study. When presented with potentially thousands of publications it can be difficult to find existing publications in a given research area, and when relevant publications have been found, lack of usage information (e.g. in the form of download statistics, citation counts etc) can make it difficult to prioritise" pg. three

There is a disconnect between this paragraph and the one before - clearer connections between the arguments and rationale here how have the CLOSER studies led to this rationale in this paragraph.

For first time users - it would be helpful to define what you mean by, "bibliometric", as it used quite frequently in the paper.

\section{Exemplar Publication list (p4)}

You state here the exemplar list of publications considered in this project is from the Avon Longitudinal Study of Parents and Children (ALSPAC). It would really be helpful from the outset if the author could be clear that this was a project undertaken to test the PUMA pipeline there seems to be a switch from study to project - to maintain consistency perhaps refer to one description.

\section{Methods}

Page 7 - unclear by what you mean by 'Use Case' - what does this mean?

I found the diagram and definition of each stage very helpful in regards to the process undertaken.

\section{Discussion}

It would be really helpful to know how long it took to use PUma when trialling it for this project. Where there any technical glitches? How user friendly it is, particularly in regards to accessibility (especially for students or first time researchers)?

\section{Future work}

If this is to be utilised by others what will be available as a resource guide for those who want to use it (e.g. training etc.)?

\section{Headings and signposting}

There seem to be three types of headings being used to signpost different sections. At times I found this confusing as the main heading and subheading seem to be same font size and weight (e.g. see discussion the limitations underneath then you have sub subheadings in italics).

\section{Is the rationale for developing the new software tool clearly explained?}

Yes

Is the description of the software tool technically sound?

Yes

Are sufficient details of the code, methods and analysis (if applicable) provided to allow 
replication of the software development and its use by others?

Partly

Is sufficient information provided to allow interpretation of the expected output datasets and any results generated using the tool?

Partly

Are the conclusions about the tool and its performance adequately supported by the findings presented in the article?

Partly

Competing Interests: No competing interests were disclosed.

Reviewer Expertise: Primary Qualitative research, systematic reviews in evidence base policy in public health. Specialist in stakeholder engagement, qualitative evidence synthesis and thematic analysis. Lecture on MSc module : Introduction to Qualitative Methods.

I confirm that I have read this submission and believe that I have an appropriate level of expertise to confirm that it is of an acceptable scientific standard, however I have significant reservations, as outlined above.

\section{Author Response 26 Feb 2021}

\section{Olly Butters}

Thank you for taking the time to review this paper, and we're very happy to hear you found it interesting! I think we have addressed all of your comments in the text. Below we itemise each one and how we have addressed it.

\section{Abstract}

Added text to be explicit about this being a test of PUMA using a list of publications from a cohort study.

\section{Introduction}

Comparing to another dataset - we found that the amount of work required to generate a clean list of publications was high (see the ALSAPC data paper referenced here). For now, we just wanted to show what the pipeline is. Our plan is to do as you suggest in the future and run the pipeline on a selection of different studies so that we can look in more detail at the differences between them.

Cohort study web form searching - tidied up language, and updated text to reflect CLOSER's increased membership.

Restructured the introduction to make this clearer.

Added a bibliometrics definition

\section{Exemplar publication list}

Moved this section to the use case section and made it clearer throughout that this data is to show the pipeline working.

Tidied use of project/study throughout. 


\section{Methods}

Use case - The term use case comes from the F1000 software tool article structure and it's just meant to show an example of the pipeline running.

\section{Discussion}

Outlined in the operation section that it is a rather technical program to run, and also mentioned in limitations section. Added the time it took to run in use case section. Added discussion point about its development.

\section{Future work}

Currently, I'd expect the main users to be at the more technical/development end. The documentation on the wiki covers all aspects of how to run it and was sufficient for Leslie McIntosh to install and replicate the analysis. It would be interesting to partner with some bibliometric analysis researchers to see where the software and training needs to go next, but that will require more funding.

\section{Headings}

Tidied up use of headings to make it easier to follow.

Competing Interests: No competing interests were disclosed.

Reviewer Report 26 November 2020

\section{https://doi.org/10.5256/f1000research.28123.r72761}

(c) 2020 McIntosh L. This is an open access peer review report distributed under the terms of the Creative Commons Attribution License, which permits unrestricted use, distribution, and reproduction in any medium, provided the original work is properly cited.

\section{Leslie McIntosh}

Ripeta, Saint Louis, MO, USA

\section{Overall Comments}

Improving the search capabilities across studies based on research manuscripts is challenging and important. The authors have built a pipeline to improve the capture of metadata, thus addressing this challenge. The links to the data and software work. Moreover, I replicated the study with the available data, code, and instructions without contacting the authors. While there are many comments below, the majority pertain to improving the article readability. Great work on building this pipeline.

\section{Open Science}

This part of the review focuses on the transparency of the work looking for quality indicators that are important for a good manuscript. While all of these elements are not necessarily required by the publisher, the presence and clarity of them make your work more transparent.

\section{$\underline{\text { Authors }}$}


One author has an ORCID listed while the others do not. It is best practice to have unique, persistent author identifiers such as an ORCID for each author.

\section{Study Objective}

Your Text from the Introduction: This article describes an open source software pipeline (PUMA PUblications Metadata Augmentation pipeline) which takes a list of publications and augments it with metadata from a selection of third-party metadata providers.

Comment: This technically states what you are doing and is not a clear study objective. A clear, concise, study objective will help in reading and understanding the science (e.g., The purpose of building the software pipeline is to improve search capabilities across cohort studies through augmenting metadata.)

\section{Data Availability Statement}

The DAS is present and links to the Zenodo corpus of papers for the Use Case. You could also state that the other data presented is generated from the software/code.

Code Availability

The code is available as stated in the Software Availability statement and is working as expected.

\section{Funding Statement}

This is present with appropriate grant identifiers mentioned. I did not verify the grant IDs.

\section{Ethics Statement}

When reviewing your manuscript, I could not find the ethical approval of the research. As this appears not to apply to this research, this is acceptable.

\section{Organisation and Miscellaneous}

This section provides critiques based on the readability of the article. Where appropriate, I offer suggestions for making it more consumable to the reader.

\section{Introduction}

The information is great in the introduction, but I found it difficult to read at first. Suggestions for improvement:

The first through fifth paragraphs (ending with "The search functionality... paragraph" seems out of place. Perhaps call out the description of the cohort study data just has you have other topics in the introduction (e.g., Existing Tools, Exemplar Publication List) and put it later (see next comment).

The true introduction seems to start with the sixth paragraph "We consider the lack of a comprehensive publication..." until the 'Existing Tools' Section. This section succinctly describes the problem with searching across cohort publications and offers your study objective.

From the sentence beginning with "In this work we also make use of the persistent identifiers that the National Center for..." through the end of the next section appears to have truly started describing your Methods. 


\section{Methods}

1. The Implementation subsection is a bit challenging to read. It might make it more readable to add labels to Figure 1 so they can be referenced in each section describing it. There are invariably other possible solutions as well.

2. Describe Table 1 in more detail before it is referenced.

3. Figure 3 - the mean and median are hard to see with the yellow and green colours. (You don't necessarily need to fix this in the paper but maybe in a future iteration of the Other software.)

The keywords for the article should include something related to pipeline development and/or software.

There are a number of grammatical errors throughout the article.

Consider dropping 'etc.' when you are stating examples, especially when using '(e.g., )'

\section{Scientific Review}

This section delves into the scientific question(s) presented in the article. I am specifically looking for an interesting scientific rationale for the study as well as supporting evidence to answer the research objective(s).

Study Objective as stated in article

This article describes an open source software pipeline (PUMA -PUblications Metadata Augmentation pipeline) which takes a list of publications and augments it with metadata from a selection of third-party metadata providers. This augmented metadata set has two distinct use cases: 1) bibliometric analysis and 2) providing a web based searching and exploration tool.

Examples of the potential bibliometric analyses possible with this augmented metadata include: calculating the total number of citations a study has generated, characterising a study based on the keywords of its publications, highlighting the geographic or institutional distribution of first authors, the variety of authors, assessing which journals are published in most fre- quently, how each of these metrics is changing over time, as well as a variety of other uses. We demonstrate some of these bibliometric uses and a web based exploration tool based on the augmented metadata set provided by PUMA in this article.

1. The software ran on my local machine and using the corpus suggested within Zotero, everything replicated. Great job!

Working with Zotero does provide a few challenges, but there are notes on the GitHub wiki that walks through those challenges.

2. The counts provided Tables 2-5 come from a CSV file generated after running the code. It would be nice to have the files and code for the use case to be shared as well. This not only supports complete reproducibility, it also helps some readers understand the expected outputs without installing the software.

3. The Use Case is very nice to have in these types of projects, so I would suggest being clearer with it. What questions can you answer from these data that you could not before? Why is this important? 
4. The Discussion should loop back to the purpose of the study and restate what you achieve through your work.

Is the rationale for developing the new software tool clearly explained?

Yes

Is the description of the software tool technically sound?

Yes

Are sufficient details of the code, methods and analysis (if applicable) provided to allow replication of the software development and its use by others?

Yes

Is sufficient information provided to allow interpretation of the expected output datasets and any results generated using the tool?

Partly

Are the conclusions about the tool and its performance adequately supported by the findings presented in the article?

Yes

Competing Interests: No competing interests were disclosed.

Reviewer Expertise: Informatics, bibliometrics, scientometrics, biomedical informatics

I confirm that I have read this submission and believe that I have an appropriate level of expertise to confirm that it is of an acceptable scientific standard, however I have significant reservations, as outlined above.

Author Response 26 Feb 2021

Olly Butters

Thank you for taking the time to read the paper and installing/running it! We think we have addressed all of your comments and have itemised this below.

Open Science

ORCIDs - All authors have now added ORCIDs.

Study objective - Updated the text as suggested.

DAS - added an output section with a link to Zenodo with all the output data and some screenshots.

Organisation and Miscellaneous 
Introduction - Substantially restructured the introduction along the lines suggested here and in the other referee's comments. Some content moved into a separate background section and the use case data description moved to the use case section later on.

Method

Aligned the heading names in the implementation section to the middle column of the figure and highlighted this in the text.

Reworded how and where table 1 is referred to.

The colours on the plots are mostly configurable in the software, but I agree it is not easy to see that in the diagram. I have added the median value to the citation metrics Other table to help with this paper.

Keyword - Added 'Software pipeline'

Fixed typos and grammatical errors throughout.

Got rid of several 'etc'

\section{Scientific review}

Glad you got it to run!

Table data:

Table 2 - The counts come from manually looking at the raw data.

Table 3 - The metadata coverage table values are copied from the output HTML. I have added a screen shot in the Zenodo output data of this and it is signposted in the table caption.

Table 4 - The citation values are copied from the output HTML. I have added a screen shot in the Zenodo output data and this is signposted in the table caption.

Table 5 - Lemmatized word frequencies - Added the full list of lemmatized words to the Zenodo output data and signposted in the caption.

Use case - I have updated the text to have a brief discussion about some of the derived data.

Discussion - updated the start of the discussion to be more of a summary.

Competing Interests: No competing interests were disclosed. 
The benefits of publishing with F1000Research:

- Your article is published within days, with no editorial bias

- You can publish traditional articles, null/negative results, case reports, data notes and more

- The peer review process is transparent and collaborative

- Your article is indexed in PubMed after passing peer review

- Dedicated customer support at every stage

For pre-submission enquiries, contact research@f1000.com 